\title{
STABILITY OF FUNCTIONAL DIFFERENTIAL EQUATIONS WITH OSCILLATING COEFFICIENTS AND DISTRIBUTED DELAYS
}

\section{MiCHAEL I. GIL'}

Abstract. We consider the scalar equation

$$
\dot{x}(t)+\sum_{j=1}^{m} a_{j}(t) \int_{0}^{h} x(t-s) d r_{j}(s)=0 \quad(h=\text { const }>0, \dot{x}=d x / d t),
$$

where $r_{j}(s)$ are nondecreasing functions. Besides, we do not require that $a_{j}(t)$ are positive for all $t \geqslant 0$. So the function

$$
z+\sum_{j=1}^{m} a_{j}(t) \int_{0}^{h} e^{-z s} d r_{j}(s)
$$

can have zeros in the right-hand plane for some $t \geqslant 0$. It is proved that the considered equation is exponentially stable, provided $a_{j}(t)=b_{j}+c_{j}(t)$, where $b_{j}$ are positive constants, such that all the zeros of the function $z+\sum_{j=1}^{m} b_{j} \int_{0}^{h} e^{-z s} d r_{j}(s)$ are in the open left-hand plane, and the integrals $\int_{0}^{t} c_{j}(s) d s(j=1, \ldots, m)$ are sufficiently small for all $t>0$.

Mathematics subject classification (2010): 34K20.

Keywords and phrases: functional differential equation, linear equation, exponential stability.

\section{REFERENCES}

[1] N. V. Azbelev, P.M. Simonov, Stability of Differential Equations with Aftereffect, Stability Control Theory Methods Appl., 20, Taylor \& Francis, London, 2003.

[2] L. BerezANS Ky, E. Braverman, On exponential stability of linear differential equations with several delays, J. Math. Anal. Appl., 324 (2006), 1336-1355.

[3] L. BeREZANSKY, E. BRAVERMAN, On stability of some linear and nonlinear delay differential equations, J. Math. Anal. Appl., 314 (2006), 391-411.

[4] M. I. GIL', The Aizerman-Myshkis problem for functional-differential equations with causal nonlinearities, Funct. Differ. Equ., 11, 1-2 (2005), 175-185.

[5] A. Ivanov, E. Liz, and S. Trofimchuk, Halanay inequality, Yorke 3/2 stability criterion, and differential equations with maxima, Tohoku Math. J., 54, 2 (2002), 277-295.

[6] V. Kolmanovs KiI, A. MYshkis, Introduction to the Theory and Applications of Functional Differential Equations, Math. Appl., 463, Kluwer, Dordrecht, 1999.

[7] T. KriszTin, On stability properties for one-dimensional functional-differential equations, Funkcial. Ekvac., 34 (1991), 241-256.

[8] J. C. LiLlo, Oscillatory solutions of the equation $y^{\prime}(x)=m(x) y(x-n(x))$, J. Differential Equations, 6 (1969), 1-35.

[9] E. Liz, V. TKachenko, AND S. TRofimchuk, A global stability criterion for scalar functional differential equations, SIAM J. Math. Anal., 35 (2003), 596-622.

[10] A. D. MYSHKIS, On solutions of linear homogeneous differential equations of the first order of stable type with a retarded argument, Mat. Sbornik N.S., 28 (1951), 15-54.

[11] J. W. H. So, J. S. YU, AND M. P. CHEN, Asymptotic stability for scalar delay differential equations, Funkcial. Ekvac., 39 (1996), 1-17.

[12] X. WANG, L. LiaO, Asymptotic behavior of solutions of neutral differential equations with positive and negative coefficients, J. Math. Anal. Appl., 279 (2003), 326-338. 
[13] T. Yoneyama, On the stability for the delay-differential equation $\dot{x}(t)=-a(t) f(x(t-r(t)))$, J. Math. Anal. Appl., 120 (1986), 271-275.

[14] T. Yoneyama, On the 3/2 stability theorem for one-dimensional delay-differential equations, J. Math. Anal. Appl., 125 (1987), 161-173.

[15] T. Yoneyama, The 3/2 stability theorem for one-dimensional delay-differential equations with unbounded delay, J. Math. Anal. Appl., 165, 1 (1992), 133-143.

[16] Z. ZHANG, Z. WANG, Asymptotic behavior of solutions of neutral differential equations with positive and negative coefficients, Ann. Differential Equations, 17, 3 (2001), 295-305. 